\title{
Effect of Background Pressure on the Plasma Oscillation Characteristics of the HiVHAc Hall Thruster
}

\author{
Wensheng Huang ${ }^{*}$, Hani Kamhawi ${ }^{\dagger}$, \\ National Aeronautics and Space Administration Glenn Research Center, Cleveland, OH, 44135 \\ Robert B. Lobbia", \\ U.S. Air Force Research Laboratory RQRS, ERC Inc., Edwards AFB, CA, 93524 \\ and \\ Daniel L. Brown ${ }^{\S}$ \\ U.S. Air Force Research Laboratory RQRS, Edwards AFB, CA, 93524
}

\begin{abstract}
During a component compatibility test of the NASA HiVHAc Hall thruster, a number of plasma diagnostics were implemented to study the effect of varying facility background pressure on thruster operation. These diagnostics characterized the thruster performance, the plume, and the plasma oscillations in the thruster. Thruster performance and plume characteristics as functions of background pressure were previously published. This paper focuses on changes in the plasma oscillation characteristics with changing background pressure. The diagnostics used to study plasma oscillations include a high-speed camera and a set of high-speed Langmuir probes. The results show a rise in the oscillation frequency of the "breathing" mode with rising background pressure, which is hypothesized to be due to a shortening acceleration/ionization zone. An attempt is made to apply a simplified ingestion model to the data. The combined results are used to estimate the maximum acceptable background pressure for performance and wear testing.
\end{abstract}

$\begin{array}{ll}\text { GRC } & =\text { Glenn Research Center } \\ \text { SMD } & =\text { Science Mission Directorate } \\ \text { ISPT } & =\text { In-Space Propulsion Technology } \\ \text { HiVHAc } & =\text { High Voltage Hall Accelerator } \\ \text { EDU } & =\text { Engineering Development Unit }\end{array}$

\section{Abbreviations}

$\mathrm{MCD}=$ Mean Channel Diameter

CEX $\quad=$ Charge Exchange

SEE $\quad=$ Secondary Electron Emission

$\mathrm{CI} \quad=$ Confidence Interval

\section{Introduction}

$\mathrm{T}$ HIS paper is a continuation of a previous work on the effect of background pressure on the performance and plume of the NASA High-Voltage Hall Accelerator (HiVHAc) thruster. ${ }^{1}$ While the previous paper focused on the breakdown of performance into various efficiency factors and far-field time-averaged diagnostics, this paper will focus on plasma oscillation characteristics and time-resolved diagnostics.

The HiVHAc project is funded by the NASA Science Mission Directorate's (SMD's) In-Space Propulsion Technology (ISPT) program with the goal of delivering a Hall thruster system beyond the current state-of-the-art (SotA). The project objective is to enable various Discovery class missions while keeping the cost of the propulsion system low relative to other electric propulsion systems. Mission studies performed in 2009 showed that a $3.5-\mathrm{kW}$ HiVHAc system is able to outperform an SotA $4.5-\mathrm{kW}$ flight Hall thruster by delivering $6-12 \%$ more payload mass

\footnotetext{
${ }^{*}$ Research Engineer, In-Space Propulsion Systems, wensheng.huang@nasa.gov, Senior Member.

${ }^{\dagger}$ Research Engineer, In-Space Propulsion Systems, hani.kamhawi-1@nasa.gov, Associate Fellow.

* Senior Scientist, In-Space Propulsion Branch (AFRL/RQRS), robert.lobbia.ctr@us.af.mil, Senior Member.

${ }^{\S}$ Program Manager, In-Space Propulsion Branch (AFRL/RQRS), daniel.brown.50@us.af.mil, Senior Member.
} 
on four missions of interest. ${ }^{2}$ These missions include Vesta-Ceres rendezvous (the Dawn mission), Koppf comet rendezvous, Nereus (a near-Earth asteroid) sample return, and NEARER, which involves two near-Earth asteroid returns. The thrust profiles of the missions range from ones that favor high specific impulse to ones that favor high thrust-to-power demonstrating the wide throttle-ability of the 3.5-kW HiVHAc.

The iteration of the HiVHAc Hall thruster tested in this work is the Engineering Development Unit 2 (EDU2), which is designed to operate at up to $3.9 \mathrm{~kW}$ discharge power. This version of the HiVHAc thruster features a discharge channel replacement mechanism designed to achieve long operational life time for high specific impulse (high discharge voltage) operation. Compared to the previous iteration, which is the EDU1, the EDU2 has better thermal management, a simpler design for the channel replacement mechanism, and improved voltage isolation. The EDU2 has undergone the performance acceptance test (PAT), ${ }^{3}$ a vibration test, ${ }^{3}$ a plume divergence characterization test, ${ }^{4}$ in-situ mechanism actuation tests (unpublished), and a component compatibility test (CCT). ${ }^{5}$ The background pressure study described in this paper was performed during the CCT in April and May of 2013. ${ }^{5}$ This work produced data that can guide future HiVHAc testing, including the wear test.

To operate in a space-representative vacuum environment, the EDU2 was tested in NASA Glenn Research Center's (GRC's) Vacuum Facility 5 (VF5). Many diagnostics were deployed as a part of the testing, including a thrust stand, a retarding potential analyzer, a Wien filter, also known as an ExB probe, a Faraday probe, an infrared camera, a high-speed camera, and a set of high-speed Langmuir probes. Additional details regarding the CCT setup can be found in a publication by Kamhawi. ${ }^{5}$ The present paper will focus on setup and analysis of the high-speed camera and the high-speed Langmuir probes, as well as the implications of the background pressure study for future testing.

\section{Past Work}

Many kinds of plasma oscillations have been known to exist in Hall thrusters. ${ }^{6}$ Since the 1960's, many studies have been performed to characterize these oscillations. ${ }^{7-20}$ Yet the roles of these oscillations in relation to thruster performance and life-time are still largely unknown. Currently, there are two oscillation modes that are believed to be important to Hall thruster operations, the "breathing" mode and the "spokes" mode. Both modes are believed to be tied to ionization processes in the Hall thruster because their frequencies are in the kilohertz to tens of kilohertz range, which is most closely related to the characteristic ion and neutral transit time across the discharge axial length scale. ${ }^{6,12}$ Recently, there has been increased interest in these plasma oscillations because they have been observed to vary greatly with facility conditions ${ }^{18}$ and may be responsible for differences in thruster behavior between ground tests and on-orbit. As more Hall thrusters are being considered for flight applications, including the HiVHAc series, it has become critical for the Hall thruster community to better understand the behavior of these plasma oscillations so that on-orbit performance can be predicted using ground test data. For the present study, the high-speed camera and Langmuir probe were chosen to study these plasma oscillations.

The high-speed camera has only recently been used as a quantitative tool for studying Hall thruster plasma oscillations. High-speed optical imaging on a Hall thruster was first performed by Darnon, et. al., though the view was not perpendicular to the annulus plane and did not capture azimuthal plasma movement. The instrument Darnon, et. al., used was a charge-coupled device with an image intensifier. ${ }^{11}$ Subsequently, Lobbia, Liu, and Gallimore studied a cluster of annular Hall thrusters using a commercial high-speed camera ${ }^{21}$ while Parker, Raitses, and Fisch studied a cylindrical Hall thruster. ${ }^{22}$ Shortly after, McDonald developed a Fourier-transform technique to study both breathing and spokes mode in annular Hall thrusters. ${ }^{19}$ Sekerak then combined the Fourier-transform technique with results from high-speed Langmuir probes to study oscillation mode transition in Hall thrusters as a function of magnetic field strength. ${ }^{20}$ The analysis technique used in the present study is based on the aforementioned Fourier-transform technique.

The high-speed Langmuir probe was first used on a Hall thruster by Lobbia and Gallimore, who first identified all of the distortion effects associated with high-speed sweeping of Langmuir probes up to the megahertz range. ${ }^{23}$ Lobbia then combined a high-speed dual Langmuir probe (HDLP) with a Fourier-transform correlation technique to temporally and spatially resolve the breathing mode in a Hall thruster. ${ }^{17}$ The high-speed Langmuir probe technique used by Sekerak is a refined version of Lobbia's work. ${ }^{20}$

The breathing mode oscillation is believed to be an axial ionization wave. The name comes from the resemblance of the exhausted plasma packets to that of breaths of gas during breathing mode oscillation. The simplest quantitative description of the breathing mode oscillation is based on modeling work done by Fife. Fife started with the assumption that the breathing mode is an axial ionization-driven oscillation and derived the scaling relationship shown in Eq. (1) below. ${ }^{12}$ 


$$
2 \pi f_{i}=\frac{\sqrt{u_{i} u_{n}}}{L_{i}}
$$

In Eq. (1), $\mathrm{f}_{\mathrm{i}}$ is the frequency of the breathing mode, $\mathrm{u}_{\mathrm{i}}$ is the speed of the ions leaving the thruster, $\mathrm{u}_{\mathrm{n}}$ is the speed of the neutrals entering the ionization zone, and $\mathrm{L}_{\mathrm{i}}$ is the characteristic length over which the oscillation action is taking place. Typically, $\mathrm{L}_{\mathrm{i}}$ is taken to be the overall length of the discharge zone, where both ionization and acceleration are taking place. This scaling relationship has been shown to predict general trends in the breathing mode oscillation frequency with discharge voltage though the exact values do not always agree. ${ }^{12}$ The role of the breathing mode in electron transport is currently unclear, though Lobbia has argued that the breathing mode is an intrinsic feature in the Hall thruster and is necessary for the thruster to achieve enough electron transport to maintain the discharge. ${ }^{17}$ The scaling relation was not created with the ingestion of background neutrals in mind.

The spokes mode oscillation is believed to be an azimuthal ionization wave. The name comes from the appearance of rotating plasma packets, which resemble spokes on a wheel, in the channel of a Hall thruster when viewed from downstream of the exit plane. Works performed by Janes and Lowder ${ }^{7}$ and McDonald. ${ }^{19}$ have provided some basic understanding of the spokes mode oscillation. The spokes mode is presented as an alternate means by which electron current can be transported across magnetic field lines. Subsequent work by Sekerak showed that a Hall thruster can operate with almost no breathing mode and strong spokes mode oscillations present. Sekerak observed that the presence of spokes mode without breathing mode is correlated with high thrust efficiency for a medium-power Hall thruster. ${ }^{20}$ Spokes have been observed to travel in the same direction as the Hall current and anywhere from two to six spokes can be observed simultaneously. ${ }^{19,}{ }^{20}$ There are currently no existing scaling relationships for the number and speed of the spokes present.

\section{Experimental Setup}

The experimental setup was previously reported ${ }^{1}$ and is repeated here with new details about the high-speed diagnostics for convenience. To simplify plot labeling, operating conditions will be labeled as vvv-k.k-Pnx, where $\mathrm{vvv}$ is the discharge voltage in volts, k.k is the discharge power in kilowatts, and $\mathrm{n}$ is the normalized background pressure, defined as the number of multiples of the lowest achievable operating background pressure for the throttle point vvv-k.k. This pressure is measured by an ion gauge located directly below the thrust stand. For example P1x indicates the operating condition corresponding to the minimum achieved background pressure, while P10x indicates the background pressure is ten times that of P1x. Unless otherwise noted, all spatial positions presented in this paper have been normalized by the mean channel diameter (MCD) of the thruster. MCD is defined as the average of the inner and outer discharge-channel wall diameters.

\section{A. Test Article and Test Matrix}

The NASA HiVHAc EDU2 is a $3.9-\mathrm{kW}$ xenon Hall thruster. The thruster is highly throttle-able. The discharge voltage can vary from 200 to $650 \mathrm{~V}$, corresponding to specific impulse values of 1200 to $2700 \mathrm{~s}$. The discharge power ranges from 0.3 to $3.9 \mathrm{~kW}$. During testing, the magnetic field settings are selected to create a magnetic lens symmetric about the channel centerline, while maximizing total efficiency. This magnetic field setting optimization is performed at the lowest achievable background pressure for each throttle point. Figure 1 shows a photograph of the test equipment around the NASA HiVHAc EDU2 thruster.

For the purpose of this test, seven throttling points were selected. These seven points are 300-1.5, 300-3.0, 400-2.0, 4003.2, 500-2.0, 500-3.0, and 500-3.9. At each throttling point, the thruster settings (including magnet currents) are optimized at the lowest achievable pressure as measured by the ion gauges next to the thrust stand. Then, the background pressure was raised by injecting xenon via an auxiliary flow line that exits at $>4$ meters downstream of the thruster pointed away from the

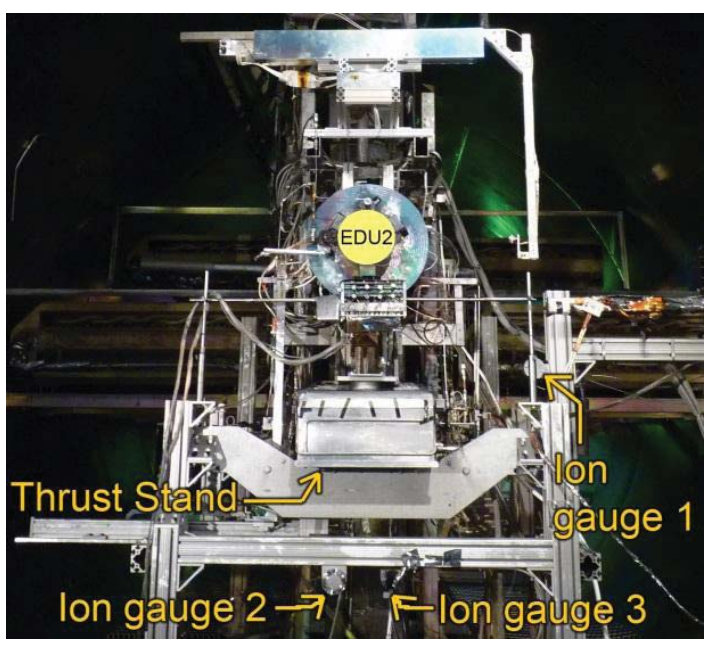

Figure 1. A photograph of the test equipment around the NASA HiVHAc EDU2 thruster. thruster. 


\section{B. Test Facility}

Testing was conducted in NASA GRC's VF5. This cylindrical facility is $4.6 \mathrm{~m}$ in diameter, $18.3 \mathrm{~m}$ long, and is pumped with cryo-panels and 20 oil diffusion pumps. The thruster was mounted on a thrust stand located close to the cryo-panels, with the thruster firing away from the panels. Facility pressures were monitored with four ion gauges, three of which were mounted next to the thrust stand and the fourth mounted on the facility wall at the same axial location as the thruster. Manufacturer specifications state that the ion gauges are accurate to $\pm 6 \%$ of reading. The positions of the gauges are shown in Fig. 1. Ion gauges 1 and 2 are both facing downstream while ion gauge 3 is facing upstream. Ion gauge 1 and 2 agree to within $10 \%$ of each other. Ion gauge 3 reports 0.63 to 0.72 times the reading of ion gauge 2. Ion gauge 2 readings were used to determine the number of multiples of the lowest achievable background pressure that the thruster was experiencing. The lowest pressure achieved for each tested condition, corrected for xenon, varied from $1.4 \times 10^{-6}$ to $2.2 \times 10^{-6}$ Torr.

Thruster propellant was supplied via commercially available mass flow controllers. These mass flow controllers were calibrated using xenon prior to testing. Typical uncertainty of measurement was $\pm 1.0 \%$ of reading. Researchgrade xenon was used.

Thruster electrical power was supplied via the Colorado Power Electronics (CPE) brassboard \#2 (BB\#2) power processing unit (PPU). ${ }^{24}$ This PPU is capable of supplying 200 to $700 \mathrm{~V}$ at up to $4 \mathrm{~kW}$ over the main discharge line as well as supply power to the cathode heater, keeper, and magnet electrical circuits. The PPU was located outside of the vacuum facility during testing.

\section{High-Speed Diagnostics}

The high-speed camera was a commercial device on loan from the Aerospace Corporation. To obtain a nonoblique view of the thruster channel without putting the high-speed camera into the plume a large plane mirror was deployed. The camera was positioned outside of the vacuum chamber and viewed the mirror through a window port. The camera was set to capture at a frame rate of roughly 80,000 frames per second at 256 by 256 pixels. In order to capture the cathode as well as compensate for possible misalignment during pump down the view area was larger than the discharge channel area, which took up a 100-by-100-pixels area. Figure 2 shows an overhead diagram of the positions of the mirror and camera. The view direction of the camera was actually $4^{\circ}$ off-axis rather than along firing axis due to the need to accommodate an IR camera. This small offset was considered acceptable as it produces negligible impact on the data analysis. High-speed video at varying background pressure was only collected for the throttle points 500-2.0, 500-3.0, and 500-3.9. High-speed video was obtained at only the lowest achievable pressure for the throttle points 300-1.5, 400-2.0, and 400-3.2.

The high-speed Langmuir probe array consisted of four pairs of Langmuir probes. Each pair consisted of one active probe and one null probe. Data from the null probe was used to correct for capacitance effects, which allows for accurate high-speed Langmuir probe sweeps. The entire probe array was mounted on a two-axis motion system and was well outside the plume when not in use. The four pairs of probes were divided into two groups. The upstream group consisted of two pairs set $2 \mathrm{~cm}$ apart in the azimuthal direction. The downstream group consisted of the other two pairs set $6 \mathrm{~cm}$ apart in the azimuthal direction. The axial distance between the upstream and downstream groups was $10 \mathrm{~cm}$. There were three measurement positions for the array at each thruster condition. For these three positions, the upstream probe was 10,15 , and $20 \mathrm{~cm}$ away from the thruster exit plane, respectively, and the probe tips were radially aligned with the centerline of the channel. To correlate the Langmuir probe signals

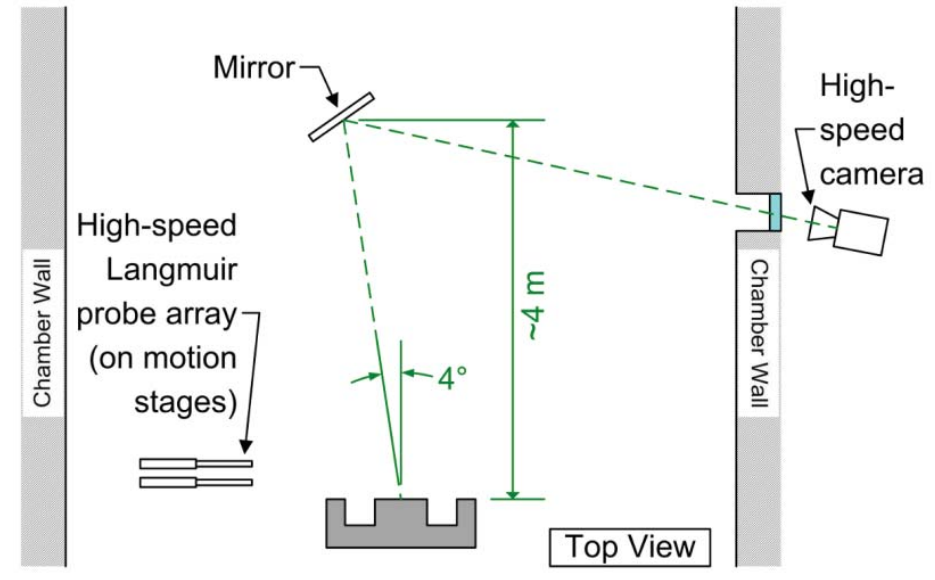

Figure 2. A diagram of the diagnostics setup.

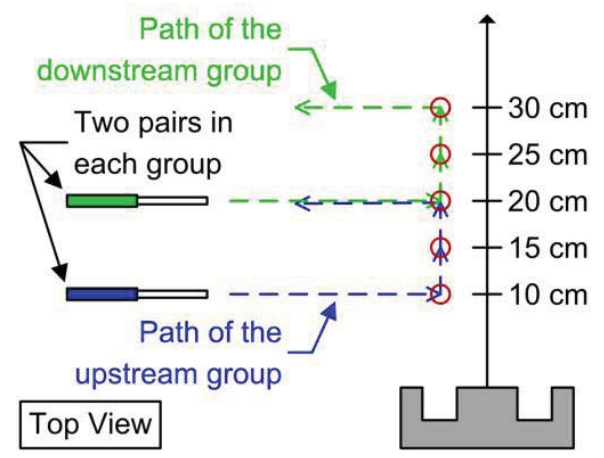

Figure 3. A diagram of the movement path of the high-speed Langmuir probe array. 
across different locations, the discharge electrical line was monitored. Figure 3 shows an overhead diagram of the movement path of the probe array and the interrogation locations with respect to the thruster.

A specialized high frequency power supply was used to supply a $200-\mathrm{kHz}$ signal through custom circuitry to sweep the probes. The custom circuitry was tuned to minimize capacitive effects associated with high-speed probe sweeping. A custom computer with high-speed data acquisition systems, high processing power, and high storage capacity is used to record the data. Figure 4 shows a photograph of the high-speed Langmuir probe array moving into place to record data while the thruster was operating.

\section{Data Analysis}

\section{A. High-Speed Camera Analysis}

For this study, the data analysis focused on the breathing and spokes mode oscillations. The high-speed camera videos were processed in the following steps.

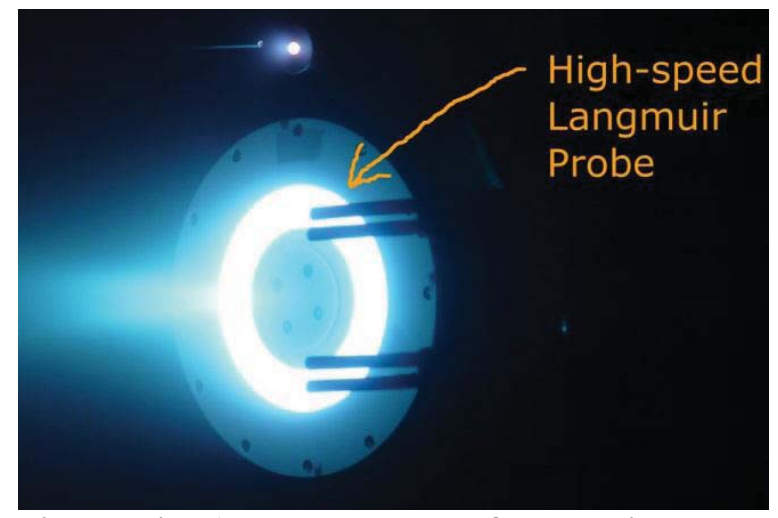

Figure 4. A photograph of the high-speed Langmuir probe array with the thruster operating. Only the downstream group of probes is visible due to the camera angle.

1. The image was cropped so that only the annulus region was analyzed.

2. The video was averaged over time to create an averaged image.

3. A circle fit was performed on the averaged image to find the center of the thruster and the boundaries of the discharge.

4. The data was divided into 120 azimuthal bins and the average intensity in each bin on each frame was calculated.

5. The binned data was normalized against the time-averaged values of the binned data.

6. A two-dimensional (2D) Fourier transform was performed to obtain a series of power spectra categorized by the mode number $\mathrm{m}$.

The data resulting from step 5 were the relative fluctuation of each azimuthal bin with time. This step produced results that are much less sensitive to variations in pixel sensitivity to light, transmission of the viewport, and reflectance of the mirror. Any breathing or spokes mode present showed up more clearly when this step was performed. Note that step 5 also removed any real, time-averaged, azimuthal variation in light intensity. For instance, the presence of a stationary hot spot would be removed by this step. This shortfall was considered acceptable because the present study was focused on oscillatory instead of stationary features. For the power spectra obtained from step 6 , the $m=0$ spectrum showed the frequency of any breathing mode present, the $m=1$ spectrum showed the frequency of any spokes mode with one spoke present, the $m=2$ spectrum showed the frequency of any spokes mode with two spokes present, and so on.

To properly interpret the power spectra from the $2 \mathrm{D}$ Fourier transform, one must first understand what the transform was really doing to the data. The transform broke down the data into a series of $2 \mathrm{D}$ sinusoids that when taken together could re-constitute the original data. Since one of the dimensions of the binned data was time and the other was azimuthal angle, the transform looked for waves that were both repeating in time and in angular space. Figure 5 shows illustrations of the ideal modal patterns that the transform was looking for when processing the data. Parts of the annulus with higher than average light intensity are shown in white while parts with lower than average light intensity are shown in black. Although the true ideal pattern have an intensity that varies in a sinusoidal fashion as opposed to having discrete black and white shades, this simplified illustration is used for better clarity.

An important feature of the modal patterns in Fig.5 is that the angular length of the light packet is necessarily shorter for higher mode number patterns than for lower mode number patterns. For example, the light packets of $\mathrm{m}$ 
$=3$ mode are 60 degrees long while the light packet of $\mathrm{m}=1$ mode is 180 degree long. Another important feature is that these model patterns have light packets that are equally spaced. However, real Hall thruster plasma waves are generally more random, with light packets that can be shorter or longer than ideal and with spacing that are not even. Thus, a spokes mode oscillation pattern with two short light packets may be de-composed into a series of modal patterns at the $\mathrm{m}=4$ mode in addition to the $\mathrm{m}=2$ mode because the length of the light packets are closer to that of the ideal $\mathrm{m}=4$ modal pattern but the spacing of the packets are more similar to the ideal $\mathrm{m}=2$ modal pattern. Another example is when 4 light spokes are present with uneven lengths and spacing, and is decomposed into a series of peaks at $\mathrm{m}=3,4$, and 5 . The real spokes pattern did not fit neatly into the ideal modal patterns but that does not necessarily mean there were 3,4 , and 5 light spokes present at the same time; instead, the real pattern is a super-positioning of $\mathrm{m}=3,4$, and 5 modal patterns on top of each other. To complicate matters further, if the thruster changes from an ideal $\mathrm{m}=3$ modal pattern to an ideal $\mathrm{m}=4$ modal pattern, and then changes to an ideal $\mathrm{m}$ $=5$ modal pattern during the filming of the high-speed video, the resulting Fourier transform can also produce a series of peaks at $\mathrm{m}=3,4$, and 5 . Thus, close examination of the actual high-speed video is sometimes necessary to determine the exact nature of the modal pattern present.

In contrast, analysis of the breathing mode oscillation can be completed by either averaging the light intensity data across all bins and performing a 1D Fourier transform or taking the $\mathrm{m}=0$ result from the 2D Fourier transform. Both approaches are performed in the present analysis to check for consistency.

\section{B. High-Speed Langmuir Probe Analysis}

High-speed Langmuir probe analysis is described in much greater detail in Lobbia's work ${ }^{25}$ and will only be briefly summarized here. The high-speed Langmuir probe data is first corrected for capacitive effects associated with high-speed sweeping. This is done by subtracting the null probe signal from the active probe signal. The data is then chopped into individual sweeps and analyzed using standard collisionless non-magnetized thin-sheath Langmuir probe theory. ${ }^{26}$ Fourier transforms are performed on the resulting number density as a function of time to obtain power spectra.

\section{Results}

\section{A. High-Speed Camera Results}

The discharge of the HiVHAc EDU2 was found to be dominated by breathing mode oscillations. For the $500 \mathrm{~V}$ throttle points at all test background pressures, there were no discernable signs of spokes mode oscillations. Spokes were found at other throttle points but were found to exist only within limited sectors around the thruster.

Figures 6-9 show the theta-t diagrams (also called spokes surfaces) for the 500-3.9-Pnx operating conditions. The theta-t diagram is a surface contour plot where the $\mathrm{x}$-axis is the azimuthal axis and the $\mathrm{y}$-axis is the time axis. Red indicates higher-than-average light intensity and blue indicates lower-than-average light intensity. Theta, the azimuthal coordinate, is defined as 0 degrees at the top of the annulus. Increasing theta is equivalent to clockwise movement on the image. Note the video is filmed through a mirror so the filmed image is a horizontally-mirrored image of the real thruster face and the reported theta coordinate is not corrected for this reflection.

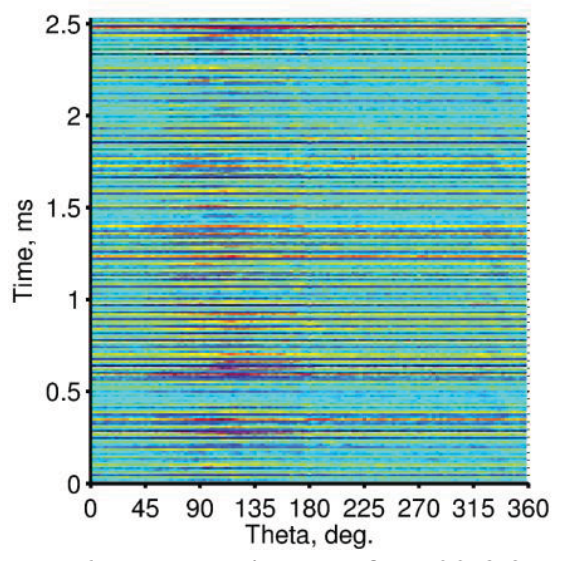

Figure 6. Theta-t diagram for 500-3.9-P1x.

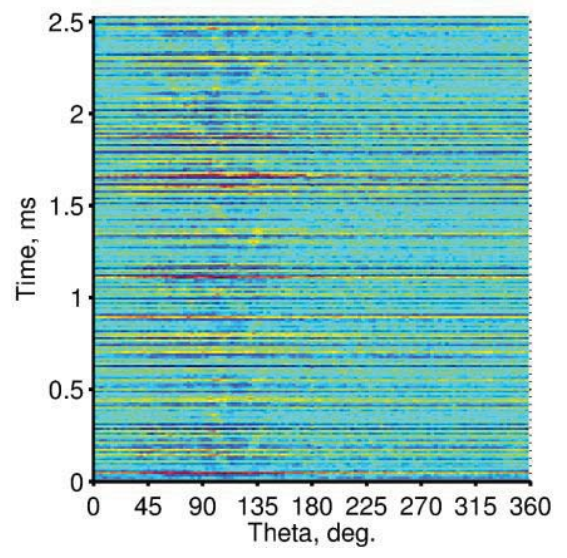

Figure 7. Theta-t diagram for 500-3.9-P3x. 


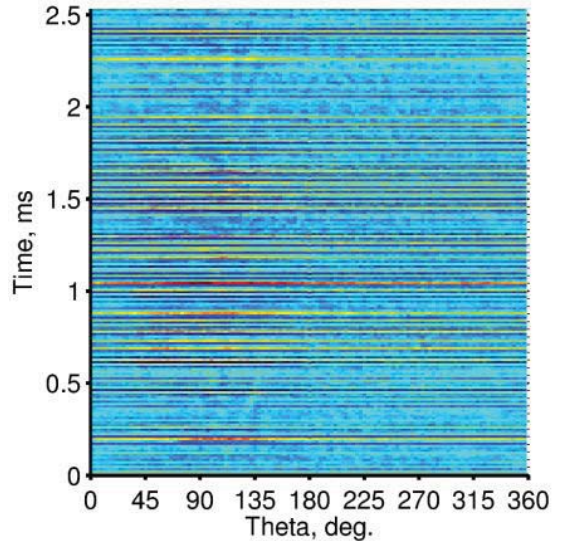

Figure 8. Theta-t diagram for 500-3.9-P5x.

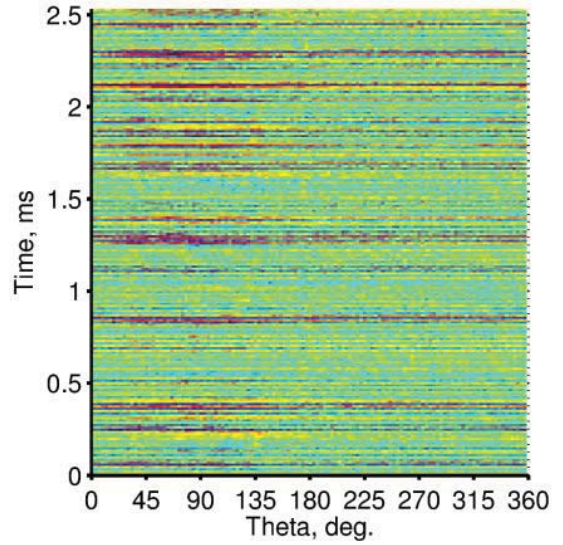

Figure 9. Theta-t diagram for 500-3.9-P10x.

From Figs. 6-9, one can see a large number of horizontal bands of color. These bands of color indicate a fairly uniform light intensity across all azimuthal locations that varies in time, which is the signature of breathing mode oscillations. In contrast, a spokes oscillation shows up as bands of diagonal lines because the light packet is traveling across both angle and time. Examination of 500-2.0 and 500-3.0 throttle point data as functions of background pressure indicates the same trend; there is a strong breathing mode of varying frequency present but no spokes mode oscillations.

Figures 10-13 show the high-speed camera power spectra for the 500-3.9-Pnx operating conditions with mode number 0 to 5 being shown. Note $\mathrm{m}=0$ corresponds to breathing mode while $\mathrm{m}=1+$ correspond to spokes mode with different modal patterns. The $\mathrm{y}$-axis of the figures is spectral power on a logarithmic scale and $\mathrm{x}$-axis is frequency in kilohertz. Since the videos were taken at 80,000 frames per second, the maximum resolved wave frequency is $40 \mathrm{kHz}$.

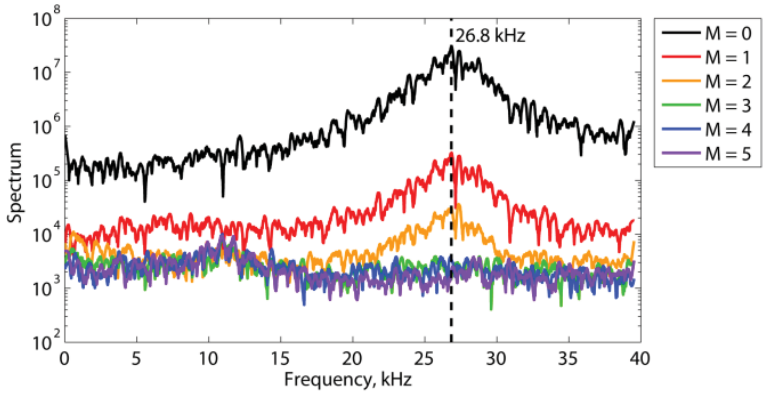

Figure 10. High-speed camera power spectra for 500-3.9-P1x.

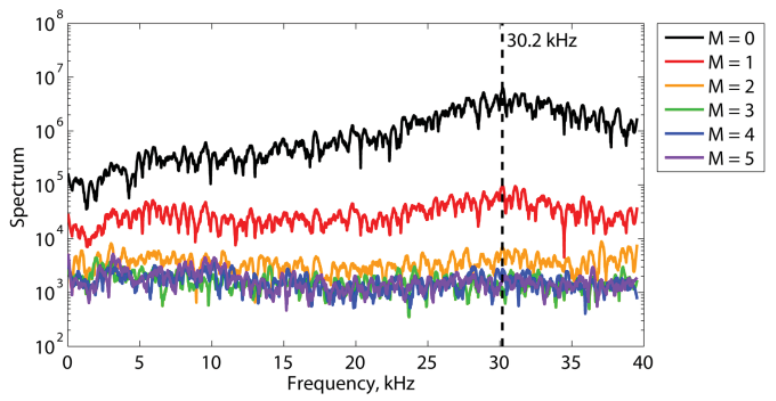

Figure 12. High-speed camera power spectra for 500-3.9-P5x.

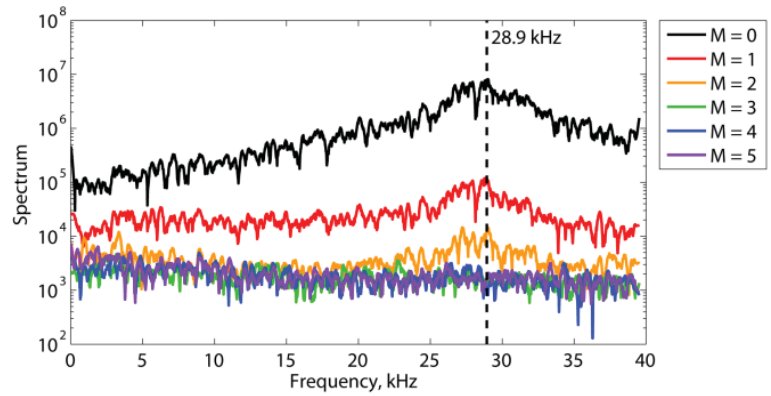

Figure 11. High-speed camera power spectra for 500-3.9-P3x.

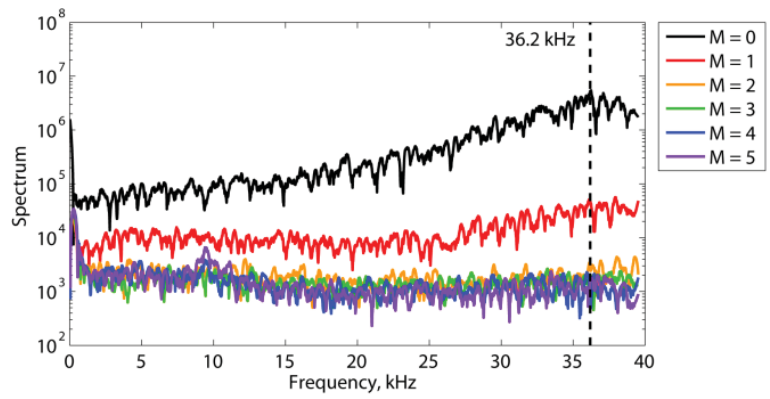

Figure 13. High-speed camera power spectra for 500-3.9-P10x.

While some peak structures can be seen for $m=1$ and 2 the peak magnitude of these structures are two orders of magnitude lower than the $\mathrm{m}=0$ peak structures. The breathing mode oscillation is clearly dominant over spokes 
mode oscillation at these operating conditions. Analysis of the 500-2.0 and 500-3.0 operating conditions show the same trend.

Although high-speed videos for the background pressure test at the $300 \mathrm{~V}$ and $400 \mathrm{~V}$ throttle points are not available, there are videos at the lowest achievable pressure that were obtained during the performance testing phase of the component compatibility test. A search through these videos was performed to help determine why spokes mode oscillation was absent in the videos of the $500 \mathrm{~V}$ operation. Examination of thruster operation at 400-3.2-P1x revealed a peculiar phenomenon where spokes mode is observed but only over a small section of the thruster annulus. Figures 14 and 15 show the theta-t diagrams and the high-speed camera power spectra, respectively, for the 400-3.2-P1x operating condition.

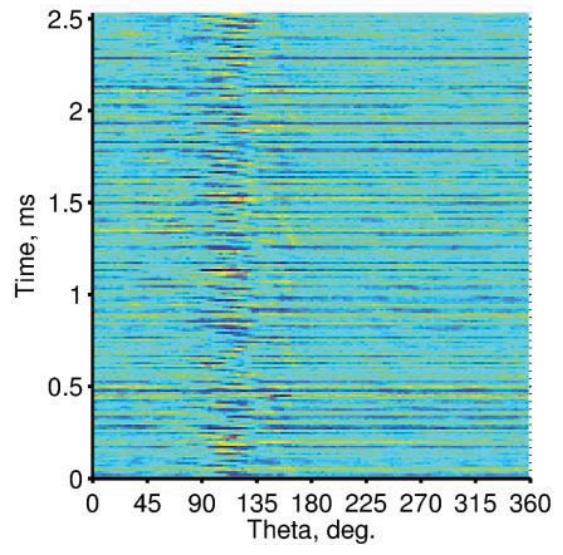

Figure 14. Theta-t diagram for 400-3.2-P1x.

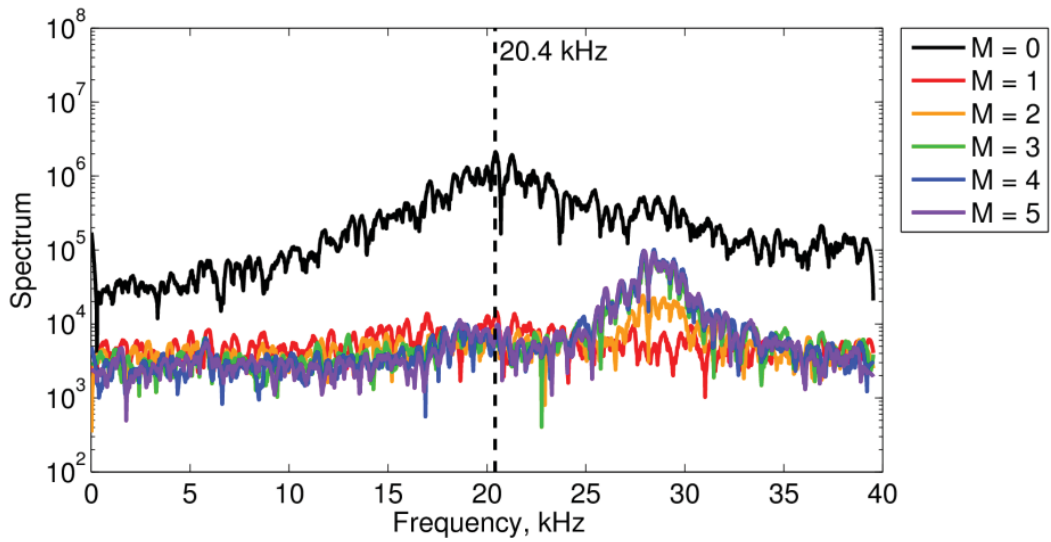

Figure 15. High-speed camera power spectra for 400-3.2-P1x.

Figure 14 indicates that a single spoke repeatedly appears and disappears in the space between theta equal to 90 and 135 degrees. The camera power spectra appear to indicate that the size of the spoke is a good match for the $\mathrm{m}=$ 4 and 5 modal patterns. The frequency of the spokes is distinctly different from that of the breathing mode oscillation. Direct observation of the high-speed video confirms the presence of this peculiar spokes mode structure. Spokes mode structure does appear to form within the HiVHAc EDU2 but some damping mechanism damps out these spokes shortly after formation. Examination of the corresponding sector of the thruster post-test did not reveal any obvious differences compared to the rest of the thruster. While it is still unclear why spokes mode oscillations could not be sustained in the HiVHAc EDU2 operating under the tested conditions, it is clear that the analysis is capable of capturing spokes, and that spokes oscillations can be formed. The spokes oscillations are just not being sustained.

Table 1 summarizes the breathing mode frequency and magnitude as functions of facility background pressure calculated from the high-speed videos. Oscillation magnitude is reported in the form of relative deviation in light intensity with a $95 \%$-confidence-interval $(95 \%$-CI), much like how relative uncertainty is typically reported. The relative deviation is reported as a percentage of the time-averaged light intensity, where $95 \%$ of the time the light intensity falls within the reported boundary. For example, if $50 \%$ relative deviation is reported, that means $95 \%$ of the time, the light intensity fell within $\pm 50 \%$ of the average light intensity. This table shows that the breathing mode frequency rises while the relative deviation of light intensity falls with rising background pressure. Recall from Eq. (1) the breathing mode frequency is theorized to scale with the square-root of the ion and neutral velocity and the

Table 1. Summary of breathing mode frequency calculated from high-speed imaging data.

\begin{tabular}{|c|c|c|c|c|c|c|c|c|c|}
\hline \multirow{2}{*}{$\begin{array}{c}\text { Disch. } \\
\text { voltage, } \\
\text { V }\end{array}$} & \multirow{2}{*}{$\begin{array}{l}\text { Disch. } \\
\text { power, } \\
\text { kW }\end{array}$} & \multicolumn{4}{|c|}{$\begin{array}{c}\text { Breathing mode frequency, } \\
\mathrm{kHz}\end{array}$} & \multicolumn{4}{|c|}{$\begin{array}{l}\text { Relative deviation of light } \\
\text { intensity, } 95 \% \text {-CI }\end{array}$} \\
\hline & & $1 x$ & $3 x$ & $5 x$ & $10 x$ & $1 x$ & $3 x$ & $5 x$ & $10 \mathrm{x}$ \\
\hline 300 & 1.5 & 9.4 & - & - & - & $16 \%$ & - & - & - \\
\hline 400 & 2.0 & 13.0 & - & - & - & $13 \%$ & - & - & - \\
\hline 400 & 3.2 & 20.4 & - & - & - & $14 \%$ & - & - & - \\
\hline 500 & 2.0 & 22.0 & 24.0 & 25.7 & - & $47 \%$ & $28 \%$ & $25 \%$ & - \\
\hline 500 & 3.0 & 20.6 & 29.2 & 29.5 & 34.4 & $36 \%$ & $31 \%$ & $32 \%$ & $23 \%$ \\
\hline 500 & 3.9 & 26.8 & 28.9 & 30.2 & 36.2 & $42 \%$ & $29 \%$ & $29 \%$ & $23 \%$ \\
\hline
\end{tabular}

American Institute of Aeronautics and Astronautics 
inverse of the characteristic length. At a given throttle point, retarding potential analyzer data previously reported shows that the ion velocity remains roughly constant as background pressure varies. ${ }^{1}$ The neutral velocity scales with the square root of the anode temperature and is expected to remain roughly constant as background pressure varies. The implication is that the breathing mode frequency must be rising with background pressure because the characteristic length is decreasing.

\section{B. High-Speed Langmuir Probe Results}

Analysis of the high-speed Langmuir probe data focused on the breathing mode oscillations. Probe measurements taken at different axial locations are generally consistent in terms of measured oscillation frequency. Electron density and temperature are observed to decrease with axial distance while plasma potential asymptotes to low tens of volts. Only plasma data from the position $10 \mathrm{~cm}$ axially downstream of the exit plane will be reported since the whole data set is too large to show here. The important trends with background pressure can be observed with only the 10-cm-axial-position data. Data from both of the probe pairs at this position gave the same answer to within measurement uncertainty and the averaged values are presented.

Table 2 summarizes the time-averaged electron density, electron temperature, and plasma potential at different operating conditions. The data are color-coded by pressure level for convenience. Generally speaking, the electron density increases with rising background pressure, which is expected if ingestion is occurring. Electron temperature and plasma potential both decrease with rising background pressure, suggesting a recession of the ion acceleration zone into the channel of the thruster.

Table 3 summarizes the frequency of the plasma oscillation and the magnitudes of oscillations in electron density and electron temperature. Oscillation magnitude is reported in the form of relative deviation with a 95\%confidence-interval. The oscillation frequencies of the electron density and electron temperature were found to be the same so only one frequency is reported. Some relative deviations of the electron temperature are greater than $100 \%$, which usually happens when there are periodic spikes in the electron temperature. This may have been an artifact of the data analysis routine or noise in the data. Despite this issue, the overall qualitative trends in the relative deviation of the electron temperature appear to be very consistent across throttle points.

The oscillation frequency of the local plasma around the probe was found to be in fairly good agreement with that of the global plasma as measured by the high-speed video. This agreement gives us some certainty that the two diagnostics are both interrogating the same breathing mode phenomenon. The frequency calculated from the high-

Table 2. Summary of time-averaged plasma properties measured by the high-speed Langmuir probe at $10 \mathrm{~cm}$ axially downstream of the exit plane along the thruster centerline.

\begin{tabular}{|c|c|c|c|c|c|c|c|c|c|c|c|c|c|}
\hline \multirow{2}{*}{$\begin{array}{c}\text { Disch. } \\
\text { voltage, } \\
\text { V }\end{array}$} & \multirow{2}{*}{$\begin{array}{l}\text { Disch. } \\
\text { power, } \\
\text { kW }\end{array}$} & \multicolumn{4}{|c|}{ Electron density, $\mathrm{m}^{-3}$} & \multicolumn{4}{|c|}{$\begin{array}{c}\text { Electron temperature, } \\
\mathrm{eV}\end{array}$} & \multicolumn{4}{|c|}{ Plasma potential, $\mathrm{V}$} \\
\hline & & $\mathbf{1 x}$ & $3 x$ & $5 x$ & $10 x$ & $1 x$ & $3 x$ & $5 x$ & $10 x$ & $1 \mathrm{x}$ & $3 x$ & $5 x$ & $10 x$ \\
\hline 300 & 1.5 & $6.4 \mathrm{e} 16$ & $6.8 \mathrm{e} 16$ & - & $7.1 \mathrm{e} 16$ & $\overline{10.1}$ & $\overline{7.8}$ & - & 4.9 & 3 & 39 & - & 24 \\
\hline 300 & 3.0 & $1.3 \mathrm{e} 17$ & $1.4 \mathrm{e} 17$ & - & $1.8 \mathrm{e} 17$ & 5.5 & 5.0 & - & 4. & 23 & 20 & - & 18 \\
\hline 400 & 2.0 & $5.3 \mathrm{e} 16$ & $6.6 \mathrm{e} 16$ & - & 7.1e16 & 10.6 & 9.5 & - & 6.0 & 54 & 43 & - & 27 \\
\hline 400 & 3.2 & $8.9 \mathrm{e} 16$ & $9.4 \mathrm{e} 16$ & - & $1.1 \mathrm{e} 17$ & 7.7 & 6.4 & - & 4.5 & 40 & 32 & - & 21 \\
\hline 500 & 2.0 & - & $3.4 \mathrm{e} 16$ & $3.9 \mathrm{e} 16$ & - & - & 7.4 & 7.0 & - & - & 36 & 32 & - \\
\hline 500 & 3.0 & $3.0 \mathrm{e} 16$ & $5.4 \mathrm{e} 16$ & $6.1 \mathrm{e} 16$ & $7.2 \mathrm{e} 16$ & 7.7 & 6.3 & 5.1 & 3.7 & 40 & 33 & 27 & 20 \\
\hline 500 & 3.9 & $5.4 \mathrm{e} 16$ & $7.2 \mathrm{e} 16$ & $8.0 \mathrm{e} 16$ & $8.9 \mathrm{e} 16$ & 7.5 & 5.6 & 4.6 & 3.5 & 38 & 30 & 25 & 18 \\
\hline
\end{tabular}

Table 3. Summary of time-resolved plasma properties measured by the high-speed Langmuir probe at $10 \mathrm{~cm}$ axially downstream of the exit plane along the thruster centerline.

\begin{tabular}{|c|c|c|c|c|c|c|c|c|c|c|c|c|c|}
\hline \multirow{2}{*}{$\begin{array}{c}\text { Disch. } \\
\text { voltage, } \\
\text { V }\end{array}$} & \multirow{2}{*}{$\begin{array}{l}\text { Disch. } \\
\text { power, } \\
\text { kW }\end{array}$} & \multicolumn{4}{|c|}{$\begin{array}{l}\text { Relative deviation in } \\
\text { electron density, } \mathbf{m}^{-3}\end{array}$} & \multicolumn{4}{|c|}{$\begin{array}{c}\text { Relative deviation in electron } \\
\text { temperature, } \mathrm{eV}\end{array}$} & \multicolumn{4}{|c|}{ Oscillation frequency, $\mathrm{kHz}$} \\
\hline & & $1 x$ & $3 x$ & $5 x$ & $10 \mathrm{x}$ & $1 x$ & $3 x$ & $5 x$ & $10 x$ & $1 \mathrm{x}$ & $3 x$ & $5 x$ & $10 x$ \\
\hline 300 & $\overline{1.5}$ & $35 \%$ & $\overline{50 \%}$ & - & $\overline{61 \%}$ & $26 \%$ & $\overline{58 \%}$ & - & $\overline{127 \%}$ & $\overline{10.1}$ & $\overline{10.7}$ & - & $\overline{11.2}$ \\
\hline 300 & 3.0 & $54 \%$ & $60 \%$ & - & $81 \%$ & $183 \%$ & $227 \%$ & - & $303 \%$ & 29 & 37 & - & 40 \\
\hline 400 & 2.0 & $37 \%$ & $44 \%$ & - & $63 \%$ & $31 \%$ & $44 \%$ & - & $122 \%$ & - & 33 & - & 38 \\
\hline 400 & 3.2 & $51 \%$ & $59 \%$ & - & $64 \%$ & $56 \%$ & $95 \%$ & - & $212 \%$ & 30 & - & - & 39 \\
\hline 500 & 2.0 & - & $32 \%$ & $35 \%$ & - & - & $29 \%$ & $37 \%$ & - & - & 25 & 31 & - \\
\hline 500 & 3.0 & $59 \%$ & $34 \%$ & $37 \%$ & $41 \%$ & $23 \%$ & $36 \%$ & $47 \%$ & $138 \%$ & 22 & 28 & 30 & 31 \\
\hline 500 & 3.9 & $38 \%$ & $37 \%$ & $43 \%$ & $53 \%$ & $27 \%$ & $40 \%$ & $72 \%$ & $220 \%$ & 30 & 29 & 30 & 36 \\
\hline
\end{tabular}

American Institute of Aeronautics and Astronautics 
speed video is of higher statistical significance because it was calculated from data around the entire annulus as opposed to a single location. Interestingly, the relative deviation in electron density and electron temperature generally increases with rising background pressure. This is the opposite trend as the relative deviation in light intensity from the high-speed video.

One possible explanation is that the plasma downstream of the acceleration zone (where the Langmuir probes are) really does behave differently with changing background pressure than the plasma inside of the acceleration zone. Note that the high-speed camera signal is dominated by light emissions from regions of the plasma with high electron temperature, which is inside the ionization and acceleration zones of the thruster where electron temperature reaches tens of $\mathrm{eV}$. Assuming the behavior of the plasma inside the channel differs from that of the plasma outside the channel, the combined results of the high-speed camera and Langmuir probes suggests a very interesting scenario. As the background pressure rises, the downstream boundary of the acceleration zone of the thruster is being pushed upstream, which is correlated with an increase in damping of the breathing mode magnitude inside the channel. At the same time, breathing mode magnitude outside the channel becomes less damped. If we further assume that the breathing mode oscillation enhances electron transport, the present data can be interpreted to mean that the shortening of the ionization and acceleration zones at high pressure reduces the amount of transport enhancement needed inside the channel (fewer magnetic field lines to transport across). At the same time, the increased distance between cathode and the downstream boundary of the acceleration zone at high pressure increases the transport enhancement needed to maintain the same flow of electrons to the channel. While this theory is consistent with the data, further experiments are needed to prove or disprove this theory.

\section{Discussions}

\section{A. Correlating Breathing Mode Frequency to Wear Mechanics and Wear Test Recommendations}

Conclusions about the change in the energy distribution of the ions bombarding the wall of the discharge channel as a function of background pressure cannot be made from the present study. However, one can correlate the breathing mode data to the length of the ionization/acceleration zone of the thruster and generate recommendations for testing from the results.

As previously stated, assuming the scaling in Eq. (1) is correct, the breathing mode frequency only scales with ion velocity, neutral velocity, and characteristic length. For a given throttle point the ion and neutral velocities are fixed so the measured breathing mode frequency is an indirect measure of the characteristic length. Since the characteristic length is roughly the length of the ionization/acceleration zone, one can indirectly measure the relative change in the length of the ionization/acceleration zone as a function of background pressure. For a thruster like the HiVHAc EDU2, the part of the ionization/acceleration zone that is inside the discharge channel is roughly the same as the erosion zone. With a reasonable assumption about how the upstream edge of the ionization/acceleration zone is anchored, the breathing mode frequency can be used to determine how the upstream edge of the erosion band is shifting (the downstream edge is at the exit plane of the thruster). This information can be used to establish an upper limit on acceptable background pressure for which the change in erosion band length is considered acceptable. The same information can also be used to quantify the change in erosion band length.

Figure 16 shows a plot of the normalized length scale as a function of normalized background pressure generated from high-speed camera data. Normalized length scale is the ratio of calculated length scale to the predicted length scale at zero background pressure. For example, a normalized length scale of 0.8 means that the calculated length scale is $80 \%$ as long as the length scale at zero background pressure. This figure shows a very consistent decrease in length scale as the background pressure is raised.

To establish a background pressure limit for future test, a few assumptions will be made. For a typical Hall thruster, the acceleration/ionization zone length is a few tens of millimeters. ${ }^{18,27,28}$ Assuming a few millimeters change in the acceleration/ionization zone length correspond to a few millimeters change in the erosion band length and assuming the design margin on the erosion band length was chosen to be a few millimeters, one can establish 0.9 as the limit for how much change in normalized length scale is considered acceptable. Using Fig. 16, the normalized pressure

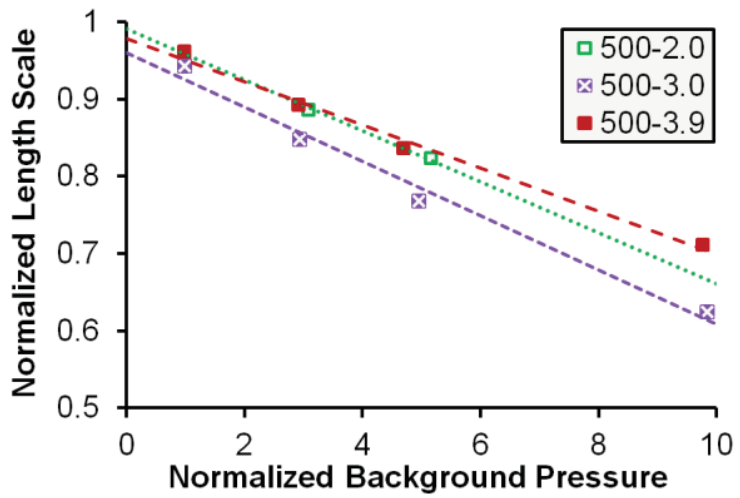

Figure 16. Normalized length scale calculated from high-speed video versus normalized background pressure. 
corresponding to a normalized length scale of 0.9 is about 2-3. Normalized pressure of 2.5 translates to about $1.2 \mathrm{e}-6$ $\mathrm{Torr} /(\mathrm{mg} / \mathrm{s})$. In other words, the erosion band length will have changed by at most a few millimeters if the operating pressure is less than $1.2 \mathrm{e}-6 \mathrm{Torr} /(\mathrm{mg} / \mathrm{s})$. Note that this criterion does not account for changes in plasma energy and overall erosion rate, only erosion band length. For reference, the criterion on background pressure based on a maximum of $2 \%$ increase in thrust due to ingestion is $5 \mathrm{e}-7 \mathrm{Torr} /(\mathrm{mg} / \mathrm{s}){ }^{1}$

\section{B. Updated Ingestion Model}

In a previous work, a two-stream ingestion model was proposed to account for ingestion of background neutrals in a Hall thruster. ${ }^{1}$ The part of the model that predicts the properties of the external stream turned out to yield nonphysical results leading to the conclusion that linearly super-imposing the internal (from propellant manifold) and external (from facility) streams on top of each other does not accurately model the ingestion physics. ${ }^{1}$ Nevertheless, calculating the ingestion rate using the change in discharge current should be a valid approach since every ingested neutral will produce an electron upon ionization. The ion will be accelerated through a part of the potential difference between the anode and the cathode, and the electron will travel the other part of the potential on its way to the anode. Figure 17 shows an illustration of the two-stream ingestion

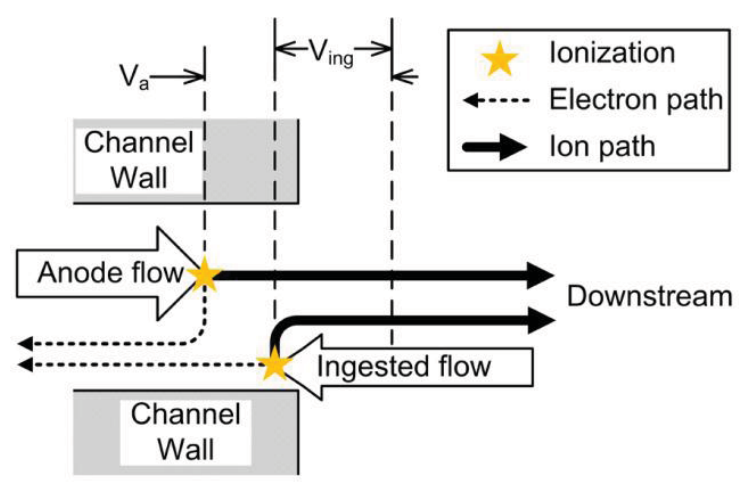

Figure 17. Illustration of the simplified twostream ingestion model. $V_{a}$ and $V_{\text {ing }}$ indicate voltages, not distances. model. For the present study, the model is modified so that the ingestion rate can be calculated without making any assumptions about the ingestion area. In the previous study, the ingestion area was assumed to be the same for all background pressures at each throttle point. ${ }^{1}$

The updated two-stream ingestion model still assumes two streams of neutrals. The first stream comes from the propellant manifold while the second stream comes from the facility. The streams have the same species composition, propellant utilization efficiency, and current utilization efficiency. Under these assumptions, the ratio of discharge current to mass flow rate for the ingested stream can be assumed to be equal to that of the main propellant stream. This ratio can be calculated using performance data from the component compatibility test. The discharge current is then plotted against the background pressure and a linear fit is performed. Zero-backgroundpressure performance of the thruster and ingested current can then be calculated. Ingested mass flow is then calculated from the ingested current using the aforementioned discharge-current-to-mass-flow-rate ratio. Table 4 shows the measured thrust, discharge current, and calculated ingestion rate. Note that one entry in the calculated ingestion is negative. This unphysical data point is likely the result of a poor curve-fit or measurement uncertainty. Recall the accuracy of the mass flow controller is $\pm 1 \%$ of reading.

Table 4. Summary of ingestion rate calculation.

\begin{tabular}{cccccccccccccc}
\hline $\begin{array}{c}\text { Disch. } \\
\text { voltage, }\end{array} \begin{array}{c}\text { Disch. } \\
\text { power, } \\
\mathbf{V}\end{array}$ & $\mathbf{4}$ & \multicolumn{4}{c}{ Thrust, $\mathbf{~ m N}$} & \multicolumn{4}{c}{ Discharge current, A } & \multicolumn{4}{c}{$\begin{array}{c}\text { Ingestion rate in percentage } \\
\text { of supplied mass flow rate }\end{array}$} \\
\cline { 3 - 16 } & $\mathbf{1 x}$ & $\mathbf{3 x}$ & $\mathbf{5 x}$ & $\mathbf{1 0 x}$ & $\mathbf{1 x}$ & $\mathbf{3 x}$ & $\mathbf{5 x}$ & $\mathbf{1 0 x}$ & $\mathbf{1 x}$ & $\mathbf{3 x}$ & $\mathbf{5 x}$ & $\mathbf{1 0 x}$ \\
\hline 300 & 1.5 & 85 & 90 & - & 95 & 4.95 & 5.06 & - & 5.31 & $0.5 \%$ & $2.4 \%$ & - & $6.9 \%$ \\
300 & 3.0 & 188 & 191 & - & 196 & 10.03 & 10.06 & - & 10.32 & $0.5 \%$ & $0.8 \%$ & - & $3.3 \%$ \\
400 & 2.0 & 105 & 109 & - & 110 & 5.04 & 5.26 & - & 5.24 & $-0.9 \%$ & $3.1 \%$ & - & $2.8 \%$ \\
400 & 3.2 & 172 & 175 & - & 181 & 8.02 & 8.05 & - & 8.19 & $0.3 \%$ & $0.6 \%$ & - & $2.3 \%$ \\
500 & 2.0 & 92 & 95 & 96 & - & 4.06 & 4.18 & 4.19 & - & $0.4 \%$ & $3.1 \%$ & $3.3 \%$ & - \\
500 & 3.0 & 139 & 144 & 146 & 149 & 5.99 & 6.08 & 6.13 & 6.22 & $0.0 \%$ & $1.5 \%$ & $2.3 \%$ & $3.7 \%$ \\
500 & 3.9 & 182 & 186 & 188 & 193 & 7.74 & 7.77 & 7.90 & 7.96 & $0.3 \%$ & $0.7 \%$ & $2.3 \%$ & $3.1 \%$ \\
\hline
\end{tabular}

\section{Dimensional Analysis on Rising Pressure and Shortening Ionization/Acceleration Zone}

While data from both the high-speed camera and the high-speed Langmuir probe suggests the ionization/acceleration zone is shortening and the downstream boundary is receding into the channel with rising background pressure, the root cause is unclear. A dimensional analysis was performed in an attempt to find the root cause. 
Equation (2) shows the relationship between the plasma potential and charge density in the form of Poisson's equation. In this equation, $\Phi$ is the plasma potential, $\rho$ is the charge density, and $\varepsilon_{0}$ is the permittivity of free space. The corresponding scaling equation is shown in Eq. (3). Note that the Laplacian of $\Phi$ scales as $V / L^{2}$ where $\mathrm{V}$ is voltage and $\mathrm{L}$ is length scale.

$$
\begin{gathered}
\nabla^{2} \Phi=-\rho / \varepsilon_{0} \\
\mathrm{~L}_{\mathrm{i}} \sim \frac{1}{\sqrt{\mathrm{n}_{\mathrm{e}}}} \sim \frac{1}{\sqrt{\mathrm{MR}_{\mathrm{a}}+\mathrm{MR}_{\mathrm{i}}}}
\end{gathered}
$$

In Eq. (3), $\mathrm{L}_{\mathrm{i}}$ is the characteristic length scale, $\mathrm{n}_{\mathrm{e}}$ is the electron density, $\mathrm{MR}_{\mathrm{a}}$ is the anode mass flow rate, and $\mathrm{MR}_{\mathrm{i}}$ is the ingested mass flow rate. Figure 18 shows a plot of the normalized length scale predicted by the above dimensional scaling against ingestion percentage for the data set in the present study. This figure shows that the results underpredict the change in length scale by about one order of magnitude. A more complex approach is needed to accurately predict the change in transport that occurs with rising background pressure.

\section{Conclusions}

The present study combined high-speed camera and high-speed Langmuir probe data to present a novel picture of the effect of facility background pressure on the plasma

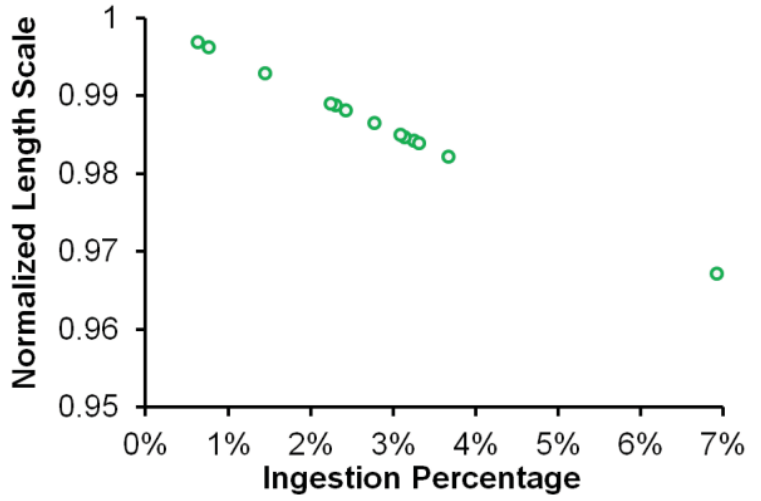

Figure 18. Predictions of normalized length scale as a function of ingestion percentage.

oscillations in the HiVHAc EDU2 Hall thruster. In the process of studying these oscillations, it was discovered that the discharge of the HiVHAc EDU2 at the operated conditions is dominated by breathing mode oscillations. Spokes can occasionally form but are quickly extinguished.

The high-speed camera data showed that the breathing mode frequency varies from 10 to $36 \mathrm{kHz}$ and tends to rise with rising background pressure. At the same time the magnitude of the light intensity oscillation inside the discharge channel falls with rising background pressure. Data from the high-speed Langmuir probe showed that the fluctuation in plasma density and temperature rises with background pressure $10 \mathrm{~cm}$ downstream of the exit plane. Data from the two diagnostics combined suggested that the ionization/acceleration zone of the thruster was shortening and receding into the discharge channel with rising background pressure. If true, the decreased characteristic plasma length would correspond to a reduction in the amount of field lines that electrons need to be transported across inside the discharge channel and may lead to a corresponding decrease in transport being provided by the breathing mode. This phenomenon would explain the decrease in the magnitude of light intensity oscillations. At the same time, the increase in distance between the downstream boundary of the acceleration zone and the cathode created an increased reliance on the breathing mode to carry the electron current from the cathode to the channel, which gave rise to an increase in fluctuation magnitude in plasma properties as seen by the Langmuir probes.

The scaling relationship between breathing mode frequency and characteristic length scale was then used to establish a maximum acceptable background pressure for which the erosion band length will have changed by at most a few millimeters. This maximum acceptable pressure was found to be $1.2 \mathrm{e}-6$ Torr $\mathrm{per} \mathrm{mg} / \mathrm{s}$ of anode mass flow rate.

An update to the simple two-stream ingestion model was made to decrease its dependency on unknown quantities. Although the model is still not capable of accurately predicting the properties of the external stream, it can be used to calculate the rough ingestion rate without constraining the effective ingestion area.

A simple dimensional analysis was performed regarding how the ingestion of background neutrals can cause the ionization/acceleration zone to shorten and recede into the discharge channel. Although the resulting prediction is qualitatively in the right direction, the prediction is off by roughly one order of magnitude. Further work is needed to determine the underlying mechanism that links rising background pressure with shortening ionization/acceleration zone.

A number of assumptions were made in the course of analyzing the data presented in this paper. The most crucial assumption was that the scaling equation for the breathing mode frequency is correct. An important next step 
will be to determine the validity of this scaling relationship. To experimentally verify the breathing mode frequency scaling relationship, one can deploy three diagnostics simultaneously. The first diagnostic will be the high-speed camera, which will be used to confirm the presence of the breathing mode and measure the associated frequency. The second diagnostic is laser-induced fluorescence velocimetry, which will be used determine the length and location of the acceleration/ionization zone as well as the velocity of the ions. The third diagnostic is either a set of thermocouples or a calibrated thermal camera to extract the anode temperature, which will be used to calculate the neutral velocity. The background pressure will then be varied at different discharge voltages and currents to produce the data needed to study the breathing mode frequency scaling relationship.

\section{Acknowledgments}

We thank the NASA Science Mission Directorate In-Space Propulsion Technology project for funding this work. We thank Eric Pencil for managing this work. We thank Edward Beiting and Rostislav Spektor of the Aerospace Corporation for lending us the high-speed camera. We thank Kevin L. Blake and George P. Jacynycz for the thruster fabrication, assembly of the test setup, and operation of the vacuum facility. And we thank Joseph Blakely of the Air Force Research Laboratory for work on the high-speed Langmuir probe.

\section{References}

${ }^{1}$ Huang, W., Kamhawi, H., and Haag, T., "Effect of Background Pressure on the Performance and Plume of the HiVHAc Hall Thruster", 33rd International Electric Propulsion Conference, 2013-058, Washington, DC, 6-10 Oct., 2013.

${ }^{2}$ Dankanich, J. W., Kamhawi, H., and Mathers, A. J., "HiVHAC Maximum Operating Power Mission Impacts", 31st International Electric Propulsion Conference, 2009-213, Ann Arbor, MI, 20-24 Sep., 2009.

${ }^{3}$ Kamhawi, H., et al., "Performance and Environmental Test Results of the High Voltage Hall Accelerator Engineering Development Unit", 48th AIAA/ASME/SAE/ASEE Joint Propulsion Conference \& Exhibit, AIAA-2012-3854, doi:10.2514/6.2012-3854, Atlanta, GA, 29 Jul.- 1 Aug., 2012.

${ }^{4}$ Huang, W., Kamhawi, H., and Shastry, R., "Farfield Ion Current Density Measurements before and after the NASA HiVHAc EDU2 Vibration Test", 48th AIAA/ASME/SAE/ASEE Joint Propulsion Conference \& Exhibit, AIAA-2012-4195, doi:10.2514/6.2012-4195, Atlanta, GA, 29 Jul.- 1 Aug., 2012.

${ }^{5}$ Kamhawi, H., et al., "Integration Test of the High Voltage Hall Accelerator System Components", 33rd International Electric Propulsion Conference, 2013-445, Washington, DC, 6-10 Oct., 2013.

${ }^{6}$ Choueiri, E. Y., "Plasma Oscillations in Hall Thrusters", Physics of Plasmas, Vol. 8, No. 4, doi:10.1063/1.1354644, Apr., 2001, pp. 1411-1426.

${ }^{7}$ Janes, G. S. and Lowder, R. S., "Anomalous Electron Diffusion and Ion Acceleration in a Low-Density Plasma", Physics of Fluids, Vol. 9, No. 6, doi:10.1063/1.1761810, 1966, pp. 1115.

${ }^{8}$ Morozov, A. I., Esipchuk, Y. V., Kapulkin, A. M., Nevrovskii, V. A., and Smirnov, V. A., "Effect of the Magnetic Field on a Closed-Electron-Drift Accelerator", Soviet Physics-Technical Physics, Vol. 17, No. 3, Sep., 1972, pp. $482-487$.

${ }^{9}$ Esipchuk, Y. V., Morozov, A. I., Tilinin, G. N., and Trofimov, A. V., "Plasma Oscillations in Closed-Drift Accelerators with an Extended Acceleration Zone", Soviet Physics-Technical Physics, Vol. 18, No. 7, Jan., 1974, pp. 928-932.

${ }^{10}$ Esipchuk, Y. V. and Tilinin, G. N., "Drift Instability in a Hall-Current Plasma Accelerator", Soviet Physics-Technical Physics, Vol. 21, No. 4, Jan., 1976, pp. 417-423.

${ }^{11}$ Darnon, F., Lyszyk, M., and Bouchoule, A., "Optical Investigation on Plasma Oscillations of SPT Thrusters", 33rd AIAA/ASME/SAE/ASEE Joint Propulsion Conference, AIAA-1997-3051, doi:10.2514/6.1997-3051, Seattle, WA, 6-9 Jul., 1997.

${ }^{12}$ Fife, J. M., Martinez-Sanchez, M., and Szabo, J., "A Numerical Study of Low-Frequency Discharge Oscillations in Hall Thrusters", 33rd AIAA/ASME/SAE/ASEE Joint Propulsion Conference, AIAA-1997-3052, doi:10.2514/6.1997-3052, Seattle, WA, 6-9 Jul., 1997.

${ }^{13}$ Gascon, N., et al., "Signal Processing and Non-Linear Behavior of a Stationary Plasma Thruster: First Results", 35th AIAA/ASME/SAE/ASEE Joint Propulsion Conference \& Exhibit, AIAA-1999-2427, doi:10.2514/6.1999-2427, Los Angeles, CA, 20-24 Jun., 1999.

${ }^{14}$ Chesta, E., Lam, C. M., Meezan, N. B., Schmidt, D. P., and Cappelli, M. A., "A Characterization of Plasma Fluctuations within a Hall Discharge", IEEE Transactions on Plasma Science, Vol. 29, No. 4, doi:10.1109/27.940951, Aug., 2001, pp. 582591.

${ }^{15}$ Litvak, A. A., Raitses, Y., and Fisch, N. J., "Experimental Studies of High-Frequency Azimuthal Waves in Hall Thrusters", Physics of Plasmas, Vol. 11, No. 4, doi:10.1063/1.1634564, Apr., 2004, pp. 1701-1705.

${ }^{16}$ Beiting, E. J., Garrett, M. L., Pollard, J. E., Pezet, B., and Gouvernayre, P., "Spectral Characteristics of Radiated Emission from SPT-100 Hall Thrusters", 29th International Electric Propulsion Conference, 2005-221, Princeton, NJ, 31 Oct. - 4 Nov., 2005.

${ }^{17}$ Lobbia, R. B., "A Time-resolved Investigation of the Hall Thruster Breathing Mode", Ph.D. Dissertation, Aerospace Engineering, University of Michigan, Ann Arbor, MI, 2009.

${ }^{18}$ Nakles, M. R. and Hargus, W. A., Jr., "Background Pressure Effects on Ion Velocity Distribution Within a Medium-Power Hall Thruster", Journal of Propulsion and Power, Vol. 27, No. 4, doi:10.2514/1.48027, Jul-Aug, 2011, pp. 737-743. 
${ }^{19}$ McDonald, M. S., "Electron Transport in Hall Thrusters", Ph.D. Dissertation, Aerospace Engineering, University of Michigan, Ann Arbor, MI, 2012.

${ }^{20}$ Sekerak, M. J., "Plasma Oscillations and Operational Modes in Hall Effect Thrusters", Ph.D. Dissertation, Aerospace Engineering, University of Michigan, Ann Arbor, MI, 2014

${ }^{21}$ Lobbia, R. B., Liu, T. M., and Gallimore, A. D., "Temporally and Spatially Resolved Measurements in the Plume of Clustered Hall Thrusters", 45th AIAA/ASME/SAE/ASEE Joint Propulsion Conference \& Exhibit, AIAA-2009-5354, doi:10.2514/6.2009-5354, Denver, CO, 2-5 Aug., 2009.

${ }^{22}$ Parker, J. B., Raitses, Y., and Fisch, N. J., "Transition in Electron Transport in a Cylindrical Hall Thruster", Applied Physics Letters, Vol. 97, No. 9, doi:10.1063/1.3486164, 1 Sep., 2010, pp. 091501.

${ }^{23}$ Lobbia, R. B. and Gallimore, A. D., "Temporal Limits of a Rapidly Swept Langmuir Probe", Physics of Plasmas, Vol. 17, No. 7, doi:10.1063/1.3449588, Jul., 2010, pp. 073502.

${ }^{24}$ Pinero, L. R., Kamhawi, H., and Drummond, G., "Integration Testing of a Modular Discharge Supply for NASA's High Voltage Hall Accelerator Thruster", 31st International Electric Propulsion Conference, 2009-275, Ann Arbor, MI, 20-24 Sep., 2009.

${ }^{25}$ Lobbia, R. B. and Gallimore, A. D., "High-speed Dual Langmuir Probe", Review of Scientific Instruments, Vol. 81, No. 7, doi:10.1063/1.3455201, Jul., 2010, pp. 073503.

${ }^{26}$ Lieberman, M. A. and Lichtenberg, A. J., Electrostatic Probe Diagnostics, in Principles of Plasma Discharges and Materials Processing, 2nd ed., Ch., doi:10.1002/0471724254, Wiley, New York, 2005, pp. 185-203.

${ }^{27}$ Huang, W., Drenkow, B., and Gallimore, A. D., "Laser-Induced Fluorescence of Singly-Charged Xenon Inside a 6-kW Hall Thruster", 45th AIAA/ASME/SAE/ASEE Joint Propulsion Conference \& Exhibit, AIAA-2009-5355, doi:10.2514/6.2009-5355, Denver, CO, 2-5 Aug., 2009.

${ }^{28}$ Huang, W., Gallimore, A. D., and Smith, T. B., "Interior and Near-Wall Ion Velocity Distribution Functions in the H6 Hall Thruster", Journal of Propulsion and Power, Vol. 29, No. 5, doi:10.2514/1.B34712, Sep-Oct, 2013, pp. 1146-1154. 